\title{
Medma Touch, Feel, Think: Survey, Catalog and Sensory Limitations
}

\author{
Franco Prampolini \\ Dina Porpiglia \\ Antonio Gambino
}

\section{Abstract}

The project is named 'Medma Touch, Feel, Think - Technological retrofit of the Archaeological Museum of Medma-Rosarno for the 3D catalog of the exibits works on display and the possible use by subjects with sensory limits'. We have carried out the 3D survey of all the main finds with analytical photomodeling techniques, their scientific cataloging on ICCD's specifications, the creation of a website with a high interactivity content and an Application that allows the sharing of extended informations for blind people, through the combined use of analog 3D models and AR authoring software.

A sustainable initiative, a driver for the technology transfer of innovation (often invoked, but rarely implemented), capable of generating 'social empowerment'. The methodology can also be shared by small entities, but characterized by contents of high historical and cultural values, expecially if they are able to build-up a territorial network of high identitarian values.

\section{Keywords}

photo-modeling, scientific cataloging, inclusion, sustainability.

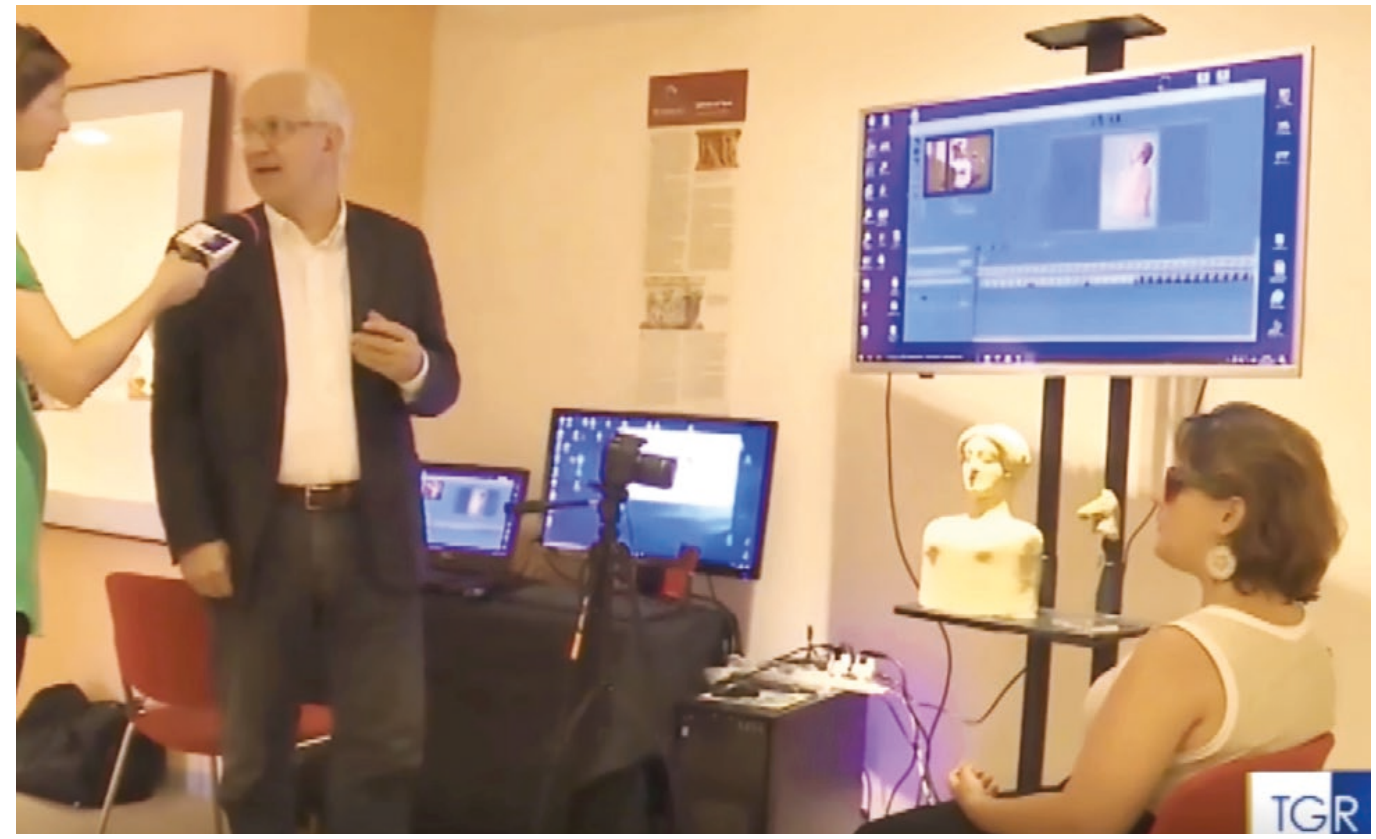


Fig. I.The MedmaRosarno Archaeological Museum in Rosarn (RC).

a. The large hall shaped like an ideal "Via Sacra" and, in the background, the s.c. "Arula Tyro". b. The large feminine terracotta busts showcase, from the sacred area of Calderazzo.

cThe "Necropolis" hall: "pano view" from the Virtual Tour materials. (Ph. Gianluca Milasi). Note the "LOGES" tactile paving path set up by the project in the museum d. The s.c. "town hall", with the findings from the Gangemi collection.
The Project rised from a proposal of the of Rotary International District 2100 (which also funded it extensively) and collects a broad institutional and social partnership: The ABAP Superintendence of Reggio C. and Vibo Valentia (a special thanks goes to Fabrizio Sudano, pro tempore manager of the Museum), the Italian Union of the Blind and Visually Impaired (UICl), The City of Rosarno, The Metropolitan City of Reggio Calabria,Terna SPA (which has effectively contributed to the financing) and many other Bodies and Associations that have contributed in different ways over time [I][2].

The Archaeological Museum of Medma-Rosarno is located in the archaeological park, in the town of Rosarno, full of olive trees that define its historical image in close connection with the actual perception of the territories, much like as they have been described directly by Paolo Orsi [Orsi 1913, passim] at the beginning of the last century on the occasion of the first major excavation campaigns. The exhibition is divided into three main sections.

It starts with the reconstruction of the necropolis: the different types of tombs are reconstructed, while 10 small showcases - intentionally shaped in such a way as to recall the cemetery "niches"- contain a small but significant sampling of the sepulchral equipment. It then continues with splendid specimens of medmean coroplastic findings - statuettes of different sizes and shapes-, busts, large masks, cryophores, vases, and iron weapons found in the sacred area of Calderazzo, presented on the sides of a virtual 'Via Sacra'. The last room contains the materials from the town, including a ritual fountain in terracotta and objects from the Giovanni Gangemi private collection, donated to the State, which consists of valuable vases with both black and red figures, including an amphora with scenes from the struggle for Achilles' arms.

The project can be summarized in five fundamental keywords.

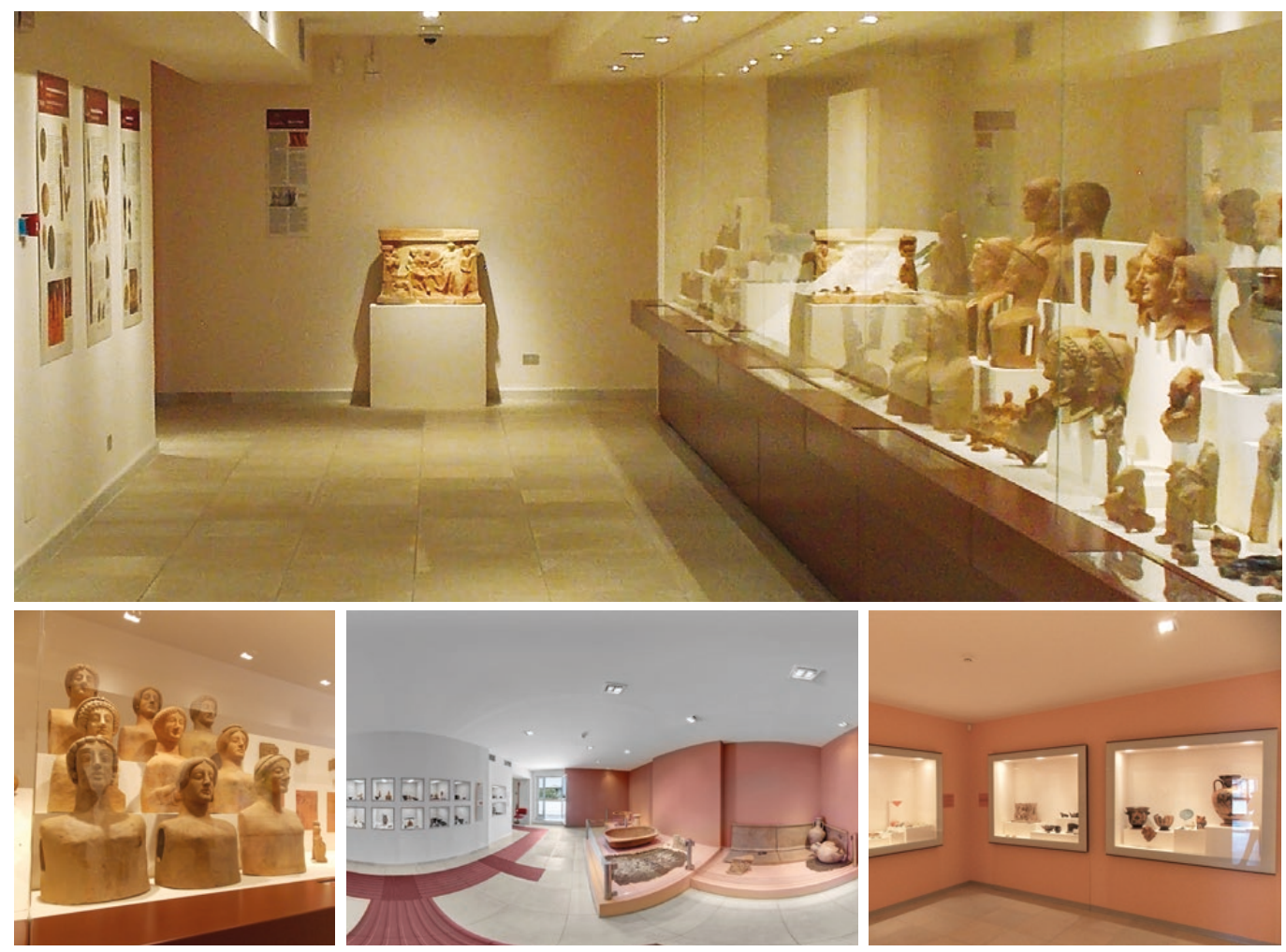

\section{Surveying and Cataloging}

Over one hundred and fifty exhibits were surveyed with analytical photo-modeling techniques, reconstructed, and scientifically cataloged in a digital environment. The results, in terms of geometric precision and chromatic accuracy, are very satisfactory. The scaling and geometric verification of the models are carried out through the use of a calibrated tablet which contains 30 markers of known coordinates singularly distinguishable by 12-bit encoding (fig. 2). The 
Fig. 2. Votive altar, (aka "Ara della Fenice") upon the calibrated grid.

Fig. 3. Catalogue form on ICCD specification.

Fig. 4. Ortoprojection and perspective section of a red figure vase. (invent. |4|437).

Fig. 5. Full color 3D print reproduction of medmean feminine bust.

Fig. 6. 3D model (front) and a study of the actual deviations between the original point and the fina mesh

"Overall surveillance" by a 5th century b.C. smal Satyr bronze. markers have different sizes, to be significant regardless of the shooting distance and to ensure rigorous verification of final residual errors. The precision obtained is firmly attested in a sub-millimeter range which makes the models themselves coherent with the $|:|$ scale survey. From the chromatic point of view - a critical factor in consideration of the particular nature of the relieved objects- excellent results were obtained thanks to a controlled shooting environment and post-production of the photos, that included the 'masking' of non-essential elements for the restitution.

The use of high-resolution cameras leads to very accurate modeling, which permits to push the analysis of single the objects to a very high level of detail, significantly expanding the possible critical data collection in fractions of time, if compared with any direct analysis, and at a very higher level of security (fig. 6).

In many occasions it has been possible to extend the survey to the entire object surface (internal/external), making it the complete 3D analysis of the object immediately available, including the direct measurement of significant points of interest straight from the model, the automatic extraction of profiles, etc. (fig. 4).

After a pre-cataloging phase based on the use of QR-Code and historical inventory numbers, an actual catalog form has been compiled, compliant with the ICCD specifications (RA-3.00) which permit direct compatibility with national databases on cultural heritage (fig. 3).
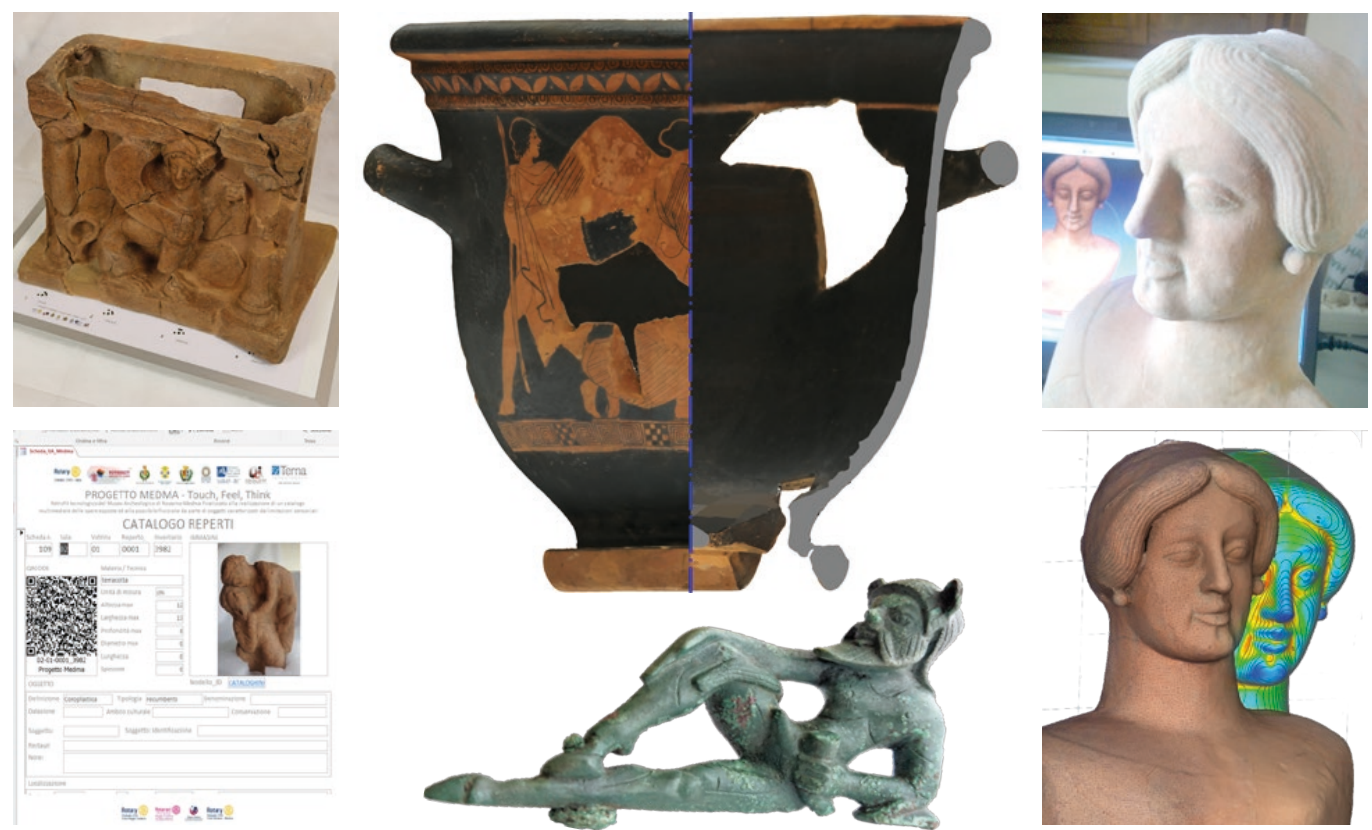

\section{$3 \mathrm{D}$ reproduction}

Three-dimensional reproductions were made on one side to, somehow, replace certain important finds transferred to other museums and, partly, to be used in an interactive application for people with sensory disabilities, both through direct full-color 3D printing in real scale and through a more 'traditional' procedure of sculpting reproduction controlled by the digital model, used for larger objects that were "oversize" for the 3D printing available technology (fig. 6). The final results, in terms of geometric precision and color accuracy, are very satisfying.

\section{Sharing and WEB}

A website has been created in a standard environment (WordPress) and permits to retrieve information about the museum, consult the multimedia catalog, and explore (or download, subject to proper authentication) the three-dimensional models at different resolution. Full-res. models in 3D/.OBJ format are available (millions of vertices point clouds, $100 \mathrm{Mb}$ or more), but we are working on smaller size models (around I $\mathrm{Mb}$ ) in the GLB format by resampling the textures to grant sharability over smartphone even at a very low band connection. We are also 
Fig. 7. Medma touch, feel, think App. From RAl TG $-\mathrm{R}$ reportage. Special thanks to RAl journalist Giulia Bondi and to Marika Meduri, president of unsighted association of Reggio Calabria (app. tester).
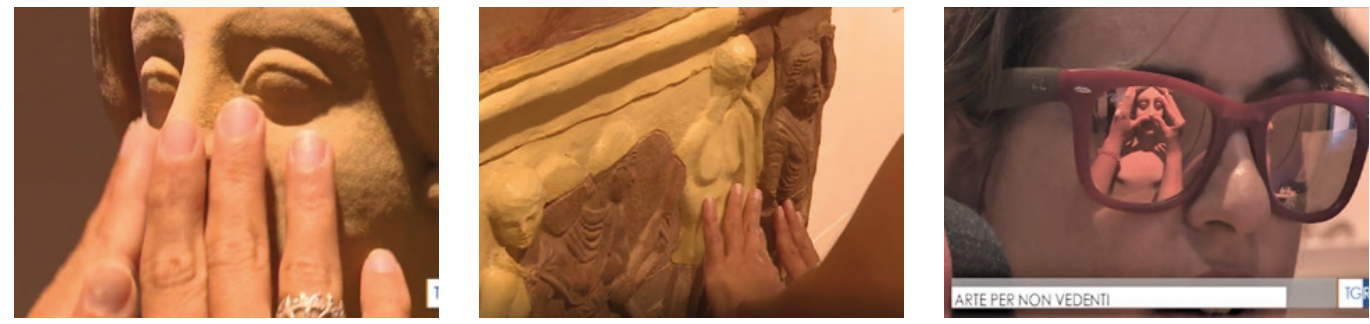

formalizing a partnership with the IIIF consortium to reach complete control over the distribution of patented 3D models. The site also provides a high level of multidisciplinary interactivity allowing specialists and scholars, through Wiki-type pages, to collaborate with the implementation of the descriptive part of the forms. Finally, it contains a complete virtual tour of the museum, which is currently being programmed to allow a direct visual consultation of the catalog.

\section{The Touch, Feel Think App}

The core commitment of the project was addressed through the implementation of an application for unsighted persons, based on the use of high-resolution webcam and motion detection techniques that allow a completely hands-free approach.

It starts with the 3D models (fig. I, 7a, 7b, 7c). People approach the replicated object and touch it without any restrictions: the system recognizes the touch and plays a first general soundtrack which introduces the historical framing of the object, and afterward, if the exploration continues, gradually recognizes the parts that are progressively touched: the description can then continue in detail, giving an account of many details that can create curiosity and encourage a scientific deepening of the knowledge of the assets, as well as their contextual conditions. The whole process is developed in an authoring environment, by which the signals coming from the cameras through the definition of any number of sensitive areas (hot-spots) can be hierarchized and can lead to a really effective storytelling program. The system also implements directional ultrasonic loudspeakers, capable of containing noise pollution in the museum by limiting the sound flow to the single users. Feedback in application testing reported a very comfortable perceptual experience. The novelty of this approach lies in the alchemical engineering of standard technologies, widely available, but not yet applied in cultural heritage environments, to achieve shareable, but very effective, results at low cost. In this case, it is of great interest the integration between motion recognition technologies with the programmability of the software capable to create a single environment for authoring multimedia contents of high semantic value, dependent on the tactile interaction between the object and the blind perceiver, thus creating a real virtual/analog bridge independent of the technological mediator on the end-user side.

\section{Conclusions}

The project is currently concluded in its prototype state and has already produced many positive feedbacks, even in this early stage, developing a profitable process that has brought together public institutions and private initiatives in a very effective synergy, characterized by a remarkable transversality.

One of the main results of this synergy was the positioning of the idea itself at the base of a further project included in the so-called 'Living-Lab Program', bottom-up initiatives financed by the European Community, directly arising from the territories as long as certain issues are perceived as strong, positive instances by the communities. A consortium has been therefore established between University and private companies for the industrialization of the prototype, within a more general enhancement action of the Museum and the Archaeological Park, that is taking shape in these very days.

The project, in this new phase, has led to the creation of an Association (ATS) between the PAU Department [3], which participates as a Research Body, and private companies active in the sector of protection, promotion, and safeguarding of cultural heritage. 
In the future, we plan to integrate into the system motion sensors with capacitive proximity devices (NFC, etc.), also through the field testing of new sensors based on the very high transduction capacity derived from the use of Graphene materials in the surface treatment of analog 3D printed models, aimed at maintaining the hands-free approach that was greatly appreciated in the testing phase in a non-immersive environment.

Beyond the specific contents of this projects, and of the new ones, we strive to combine innovation, scientific rigorous approach, and enhancement of cultural heritage through an 'inclusive attitude', where the word 'inclusion' is purified of any declination linked to the idea of the 'due by law', or, even worse, to the one of 'charitable intervention'. Cultural inclusion, and generalized fruition capabilities, on the contrary, must be intended, as they are indeed, directly connected to the economic strengthening of initiatives and territories and a powerful drive for development. This approach presents itself immediately as highly sustainable, not just being self-financed, but, thanks to the strong idea of transparent (and inclusive) commonality of all the project revenues, it can directly function as a medium for the transfer of technological innovation (often invoked, but rarely actually implemented) and generates a condition that we like to call 'social empowerment'. The entire procedure becomes immediately shareable and the methodology can also be at disposal of "limited size" cultural institutions, such as small museums, or even private collection, which are, on the other side, often bearers of the highest historical and cultural values, particularly if, together with their hosting communities, they would reach to build some territorial networks of high local identity values.

\section{Notes}

[I] Rotary International - Disctrict IT-2 I00: D.G. 20I4-20I5 prof. Giancarlo Spezie; D.G. 20I7-20I8 dott. Luciano Lucania. Rotary and Rotaract Club Reggio Calabria and Nicotera-Medma. Special thanks to: arch. Salvatore Patamia, MiBACT; arch. Pietro Vicentini (Terna); ing Giusesppe Fedele (UICI Reggio Calabria); Prof. Giuseppe Lacquaniti (Historian and Journalist).

[2] The Project has bee carried out by a group of young resources selected by public evidence from the Rotaract area and the Mediterranean University: Angela Balestrieri, Gabriele Candela, Barbara Cusato, Giuseppe Cutrupi, Roberta De Clario, Fabio Panella, Danila Punturiero, Verdiana Quattrocchi and Peppe Sorrenti. The operational coordination has been performed by Technical Tutors with consolidated experience: Antonio Gambino, Marilù Laface, Andrea Manti, and Roberto Prampolini for the web content.

[3] The 'EcoMedma' consortium consists of Ecolandia SCARL (Leading Company, Pres. Prof. Antonio Perna), The PAU Department of the Mediterranean University of Reggio Calabria (Research body, Dir. prof. Tommaso Manfredi), CADI SRL (Dir. ing. Piero Milasi).

\section{References}

Kimiko Ryokai (ed.) (2019). Augmented Reality for Visually Impaired People (AR for VIPs). University of California, Berkeley, School of Information, MIMS Capstone Project Report.

Lacquaniti Giuseppe (2014). MEDMA Colonia di Locri Epizefiri. Tropea: Romano.

Lending Mari (2018). Plaster Monuments: Architecture and the Power of Reproduction. Priceton: Princeton University Press. 7

Manduchi Roberto, Kurniawan Sri (eds.) (2017). Assistive Technology for Blindness and Low Vision. Boca Raton (USA): CRC Press.

Minto Simone, Remondino Fabio (2014). Online access and sharing of reality-based 3d models. In SCIRES-IT : SClentific RESearch and Information Technology, 4 (2), pp. 17-28.

Orsi Paolo (1913). Rosarno Medma. Esplorazione di un grande deposito di terrecotte ieratiche. In Notizie e Scavi di Antichità, suppl., pp. 55- 444.

Paoletti Maurizio, Settis Salvatore (eds.) (1981). Medma e il suo territorio. Materiali per una carta archeologica. Bari: De Donato.

Sudano Fabrizio (2019). Per un'archeologia dei culti nelle colonie locresi: gli spazi sacri di Calderazzo a Medma e del Còfino a Hipponion alla luce delle recenti scoperte. In ASNP, 5, I I/2, pp. 36-50.

\section{Authors}

Franco Prampolini, Dept. PAU - SuMMA Lab, Mediterranea University of Reggio Calabria, franco.prampolini@unirc.it Dina Porpiglia, Operational manager of the project, dina.porpiglia@gmail.com

Antonio Gambino, Dept. PAU - SuMMA Lab, Mediterranea University of Reggio Calabria, antonio.gambino@unirc.it 
